\title{
Observation of the dynamic beta effect at the Cornell Electron-Positron Storage Ring with the CLEO detector
}

\author{
D. Cinabro \\ Wayne State University, Detroit, Michigan 48202
}

M. Chadha, S. Chan, C. O’Grady, J. S. Miller, J. Urheim, A. J. Weinstein, and $F$. Würthwein

California Institute of Technology, Pasadena, California 91125

S. Prell, M. Sivertz, and V. Sharma

University of California at San Diego, La Jolla, California 92093

D. M. Asner, A. Eppich, J. Gronberg, C. M. Korte, T. S. Hill, R. Kutschke, D. J. Lange, R. J. Morrison, H. N. Nelson,

T. K. Nelson, C. Qiao, A. Ryd, D. Roberts, H. Tajima, and M. S. Witherell

University of California at Santa Barbara, Santa Barbara, California 93106

B. H. Behrens and W. T. Ford

University of Colorado, Boulder, Colorado 80309-0390

J. P. Alexander, E. Anderson, T. Banta, M. Billing, K. Bloom, S. Chapman, G. Codner, R. Cutler, D. J. Dumas,

R. Eshelman, A. D. Foland, P. Gaidarev, M. Giannella, S. Greenwald, Z. Greenwald, D. Hartill, S. Henderson, P. I. Hopman,

J. Hylas, J. Kandaswamy, N. Katayama, D. Kematick, R. Kersevan, D. L. Kreinick, D. Kubik, Y. Li, W. Liu,

R. Meller, N. B. Mistry, D. Peterson, K. Ormond, S. Peck, T. Pelaia, D. Rice, J. Rogers, G. Rouse, D. Rubin, D. Sagan, J. Sikora, M. Sloand, S. Smith, A. Soffer, R. Sproul, J. Swat, C. Tan, S. Temnykh, C. Ward, J. Welch, and E. Young Cornell University, Ithaca, New York 14853

P. R. Avery and C. Prescott

University of Florida, Gainesville, Florida 32611

R. A. Briere, D. Y.-J. Kim, and H. Yamamoto

Harvard University, Cambridge, Massachusetts 02138

T. Bergfeld, J. Ernst, G. E. Gladding, I. Karliner, M. Palmer, M. Selen, and J. J. Thaler

University of Illinois, Champaign-Urbana, Illinois 61801

\section{R. Janicek}

McGill University, Montréal, Québec, Canada H3A $2 T 8$

and the Institute of Particle Physics, Ottawa, Ontario, Canada K15 5B6

S. J. Richichi and W. R. Ross

University of Oklahoma, Norman, Oklahoma 73019

J. Fast and D. H. Miller

Purdue University, West Lafayette, Indiana 47907

S. E. Roberts

University of Rochester, Rochester, New York 114627

C. P. Jessop and K. Lingel

Stanford Linear Accelerator Center, Stanford University, Stanford, California 94309

M. Artuso

Syracuse University, Syracuse, New York 13244

V. Jain and K. W. McLean

Vanderbilt University, Nashville, Tennessee 37235

R. Godang

Virginia Polytechnic Institute and State University, Blacksburg, Virginia 24061

(Received 30 June 1997; revised manuscript received 3 September 1997) 


\begin{abstract}
Using the silicon strip detector of the CLEO experiment operating at the Cornell Electron-Positron Storage Ring (CESR), we have observed that the horizontal size of the luminous region decreases in the presence of the beam-beam interaction from what is expected without the beam-beam interaction. The dependence on the bunch current agrees with the prediction of the dynamic beta effect. This is the first direct observation of the effect. [S1063-651X(98)02501-X]
\end{abstract}

PACS number(s): 29.27.Bd

In a high energy colliding beam storage ring when two beams of oppositely charged particles pass through each other there is an attractive force between them. Under certain circumstances this beam-beam interaction can lead to a reduction in the transverse beam size at the interaction point. This "dynamic beta" effect is potentially important since a smaller beam size corresponds to a higher luminosity.

The dynamic beta effect has been long predicted [1]. It has been observed indirectly through its effect on luminosity at Cornell Electron-Positron Storage Ring (CESR) [2]. This paper describes a direct observation of the reduction in beam size due to the effect. The dynamic beta effect is analyzed by modeling the beam-beam interaction as a linear focusing lens. This focusing alters the beta, $\beta$, around the ring. The beta with the beam-beam interaction can be given in terms of the "unperturbed" $\beta_{0}^{*}$, the beta at the interaction point. For the horizontal

$$
\frac{\beta_{x 0}^{*}}{\beta_{x}^{*}}=\sqrt{1-\left(2 \pi \xi_{x}\right)^{2}+2\left(2 \pi \xi_{x}\right) \cot \left(2 \pi Q_{x 0}\right)},
$$

where $Q_{x 0}$ is the unperturbed horizontal tune and $\xi_{x}$ is the beam-beam parameter

$$
\xi_{x} \equiv \frac{\beta_{x 0}^{*} N r_{e}}{2 \pi \gamma \sigma_{x}^{*}\left(\sigma_{x}^{*}+\sigma_{y}^{*}\right)},
$$

where $N$ is the number of particles per bunch $(N=1.61$ $\times 10^{10}$ times the bunch current in $\mathrm{mA}$ at CESR), $r_{e}$ is the classical electron radius $\left(2.82 \times 10^{-15} \mathrm{~m}\right), \gamma$ is the relativistic factor $\left(1.04 \times 10^{4}\right.$ at CESR), and the $\sigma^{*}$ 's are the horizontal $(x)$ and vertical $(y)$ beam sizes at the interaction point. Note that the $\sigma^{*}$ 's are dependent upon the $\beta^{*}$ 's in the above equations. Equation (1) makes it clear that if the tune is just above the half integer resonance there is a sharp reduction in $\beta^{*}$. This decreases the size of a single beam at the interaction point, given by $\sigma^{*}=\sqrt{\beta^{*} \epsilon}$ where $\epsilon$ is the emittance. The strength of the linear lens and therefore the reduction in $\beta^{*}$ become larger with increases in the bunch current.

CESR is a particularly good place to observe the dynamic beta effect. Table I gives some of CESR's parameters and CESR is described in full detail elsewhere [3]. The bunch current is large, over $8 \mathrm{~mA}$, generating a large beam-beam interaction. There is also a large range of bunch currents during normal CESR operations as the beam lifetime is about $3 \mathrm{~h}$ and the typical fill lasts $75 \mathrm{~min}$. The collisions studied here have bunch currents ranging from 4 to $8 \mathrm{~mA}$. In the horizontal direction the CESR lattice has a tune just above the half integer contributing to a large dynamic beta effect. In the vertical direction the tune is not close to the half integer and thus dynamic beta effects are much smaller on the vertical size of the beam.
The CLEO detector has been described in detail elsewhere [4]. A recent addition is a silicon strip vertex detector [5], which is crucial to the observation of the dynamic beta effect. This consists of three layers of silicon wafers arrayed in an octagonal geometry around the interaction point. The first measurement layer is at a radius of $2.3 \mathrm{~cm}$ and the wafers are read out on both sides by strips, which are perpendicular to each other. The readout strips have a pitch of about $100 \mu \mathrm{m}$ and with charge sharing the detector has an intrinsic per point resolution of better than $20 \mu \mathrm{m}$ in both the plane transverse to the beam, the $x y$ plane, and in the direction parallel to the colliding beams, called $z$.

We observe the luminous region with events of the type $e^{+} e^{-} \rightarrow$ hadrons, which are defined as events with more than two charged tracks and four or more significant energy deposits in the electromagnetic calorimeter. All the data are taken with electron-positron collisions with a center of mass energy equal to or just below the mass of the $Y(4 S)$ resonance. We try to form a vertex from all the tracks in the event that have hits in at least two of the three layers of the silicon in both the $x y$ plane and $z$ direction. Those tracks with a contribution to the vertex chi-square of over two per degrees of freedom are removed as being unlikely to share a common vertex with the other tracks. This procedure is iterated until all such tracks are removed. Finally the probability of the vertex chi-squared must be greater than $10 \%$. Vertices formed from two or more tracks give three-dimensional points in space that are used to determine the size of the luminous region. A total of 77327 such vertices are found and used for these measurements.

For each discrete fill of CESR the average position is found in the vertical and horizontal directions. There are occasional large shifts in this average fill position that result from tuning CESR for backgrounds or luminosity with the introduction of closed orbit distortions through the interaction region. These fill averages are subtracted from all the points of the fill to correct for these shifts in the average

TABLE I. Operational parameters of CESR. IP denotes interaction point.

\begin{tabular}{lcc}
\hline \hline \multicolumn{1}{c}{ Parameter } & Symbol & Value \\
\hline Energy & $\mathrm{E}$ & $5.29 \mathrm{GeV}$ \\
Unperturbed horizontal beta at the IP & $\beta_{x 0}^{*}$ & $1.38 \mathrm{~m}$ \\
Unperturbed vertical beta at the IP & $\beta_{y 0}^{*}$ & $1.89 \mathrm{~cm}$ \\
Unperturbed horizontal emittance & $\epsilon_{x 0}$ & $2.11 \times 10^{-7} \mathrm{~m}$ \\
Unperturbed vertical emittance & $\epsilon_{y 0}$ & $2.12 \times 10^{-9} \mathrm{~m}$ \\
Bunch length & $\sigma_{z}$ & $1.87 \mathrm{~cm}$ \\
Horizontal integer tune & $Q_{x}$ & 10.540 \\
Vertical integer tune & $Q_{y}$ & 9.630 \\
Transverse damping time & $\tau_{\text {damp }}$ & $10000 \mathrm{turns}$ \\
\hline \hline
\end{tabular}




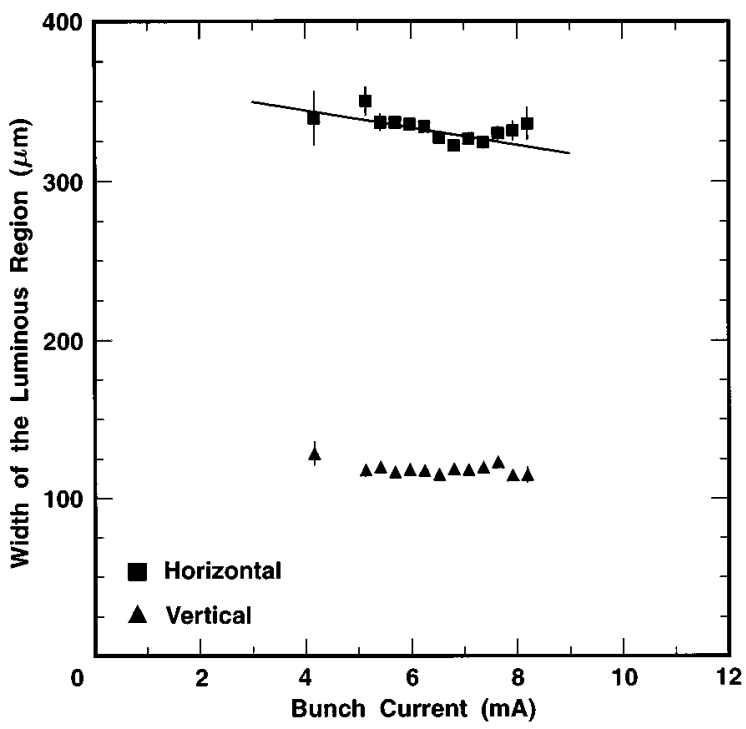

FIG. 1. The observed width of the luminous region in the horizontal and vertical directions as a function of the bunch current. Also shown is the line from the fit to the horizontal widths described in the text.

position of luminous region. We have looked for changes in the average beam position during the course of a fill and can discern none.

The distribution of these positions in the horizontal and vertical directions is binned and then fit to a flat function, to account for vertices not resulting from beam-beam collisions, plus a Gaussian shape. The width of this Gaussian is taken as the observed width of the luminous region.

Another useful feature of the CESR lattice for the observation of the dynamic beta effect is the disparity of the beam size between the horizontal and vertical directions. Without considering the effect of the beam-beam interaction the Gaussian width of the beam in the horizontal direction is $\sigma_{0 x}^{*}=540 \mu \mathrm{m}$. In the vertical direction $\sigma_{0 y}^{*} \approx 10 \mu \mathrm{m}$. Note that when the two beams collide they create a luminous region, which is the overlap of the two colliding beams. The size of this luminous region is a factor of $\sqrt{2}$ smaller than the single beam size.

Figure 1 shows the observed width of the luminous region in the horizontal and vertical directions as a function of the bunch current. The vertical width of around $120 \mu \mathrm{m}$ is much larger than the expectation of $10 / \sqrt{2}=7 \mu \mathrm{m}$. Studies with a Monte Carlo based simulation of hadronic events in the CLEO detector indicate that the vertical width of the luminous region, which is observed to be much larger than the expectation, is actually a measure of the resolution on the luminous region in the $x-y$ plane. Note that there are observations of strong beam-beam effects on the vertical beam size [6]. Also note that the bunch length is similar to the vertical size of the luminous region; thus there is an appreciable "hourglass" effect [7] in the vertical direction. These effects, and any vertical dynamic beta effects, have no impact on this observation as our resolution is much larger than the vertical size of the luminous region.

The observed horizontal width is as small as $320 \mu \mathrm{m}$ which is smaller than the expected unperturbed value of $540 / \sqrt{2}=380 \mu \mathrm{m}$. Interpreting the observed vertical width as

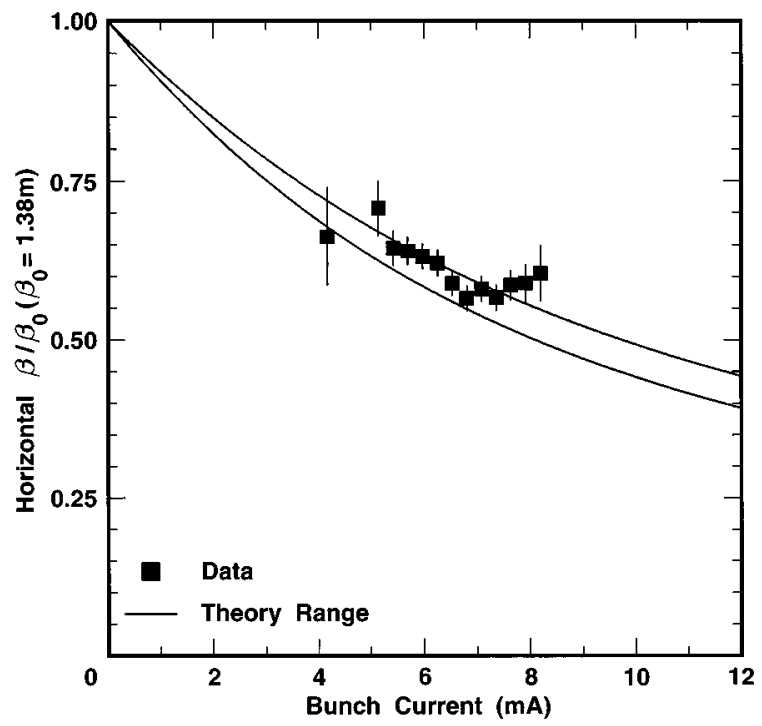

FIG. 2. The horizontal beta as a function of bunch current. Note that the error on $\beta_{x 0}^{*}$ of $5 \%$ of its value is not included in the error bars on the data.

the resolution implies an underlying horizontal width of the luminous region of $\sigma_{x \mathcal{L}}=295 \mu \mathrm{m}$, which is much below the expected unperturbed value.

If we fit the observed vertical width as a function of the bunch current to a line the width is seen to be independent of the bunch current. This is expected if the vertical width is dominated by the resolution. A linear fit to the horizontal width as a function of bunch current has a negative slope of $-5.4 \pm 1.6 \mu \mathrm{m} / \mathrm{mA}$. Both the observation of a significantly smaller than expected horizontal width of the luminous region in the presence of the beam-beam interaction and a significant dependence of the horizontal width on the bunch current are evidence of the dynamic beta effect.

To compare with the prediction of the dynamic beta effect we extract the horizontal beta from the width of the luminous region. We use a resolution of $118 \pm 10 \mu \mathrm{m}$. This is from a fit to the vertical distribution independent of bunch current with the error dominated by a $10 \mu \mathrm{m}$ jitter introduced by the correction for the average fill position. The horizontal emittance is calculated using the theoretical lattice with a thin quadrupole at the IP to simulate the beam-beam interaction. Variations in the quadrupole strength due to variations in the beam current lead to variations in the Twiss parameters around the ring. This variation leads to variation of the radiation integrals and hence to a variation of the emittance [2]. The emittance is parametrized by $\epsilon_{x}=\left(1.96 \times 10^{-7}\right.$ $\left.+1.46 \times 10^{-9} I_{\text {bunch }}+5.15 \times 10^{-12} I_{\text {bunch }}^{2}\right) \mathrm{m}$, where $I_{\text {bunch }}$ is the bunch current in $\mathrm{mA}$. We have checked this calculation of the horizontal emittance using $e^{+} e^{-} \rightarrow \mu^{+} \mu^{-}$events observed with CLEO. They give $\epsilon_{x}=(1.87 \pm 0.65) \times 10^{-7} \mathrm{~m}$, where the error is dominated by the resolution on the angle between the two muons. Then using $\beta_{x}^{*}=\sigma_{x \mathcal{L}}^{2} / 2 \epsilon_{x}$ we calculate $\beta_{x}^{*}$ as a function of the bunch current as shown in Fig. 2. The $\beta_{x 0}^{*}$ of $1.38 \pm 0.07 \mathrm{~m}$ is taken from a single beam measurement using the observed phase advance through the interaction region. 
The theory curves shown in Fig. 2 also include the small effect of the beam-beam interaction at parasitic crossings away from the interaction point where the beams are separated by a pretzel orbit. This effect serves to lower $\beta_{x}^{*}$ by about $10 \%$ at a bunch current of $12 \mathrm{~mA}$ and is essentially linear from no effect at zero bunch current. The range of the theory comes from the observed variation in the fractional integer tune from 0.537 to 0.544 from fill to fill.

In conclusion, we have directly observed the dynamic beta effect at CESR with the silicon strip detector of the CLEO experiment. We have seen that the horizontal size of the luminous region is smaller than expected when there is a beam-beam interaction, and that it decreases in size with increasing bunch current as the dynamic beta effect predicts. When we extract the horizontal beta as a function of the bunch current it is seen to agree very well with the expectation of the dynamic beta effect. This is the first direct observation of this effect.

J.P.A. thanks the NYI program of the NSF, M.S. thanks the PFF program of the NSF, M.S., H.N.N., and H.Y. thank the OJI program of DOE, M.S. and V.S. thank the A. P. Sloan Foundation, and M.S. thanks Research Corporation for support. This work was supported by the National Science Foundation, the U.S. Department of Energy, and the Natural Sciences and Engineering Research Council of Canada.
[1] B. Richter, in Proceedings of the International Symposium on Electron and Positron Storage Rings, Saclay, 1966 (Presses Universities de France, Paris, 1966); A. Chao, Physics of Collective Beam Instabilities in High Energy Accelerators, (Wiley, New York, 1993).

[2] David Sagan, in Proceedings of the 1995 Particle Accelerator Conference and International Conference on High Energy Accelerators, Dallas, 1995 (IEEE, New York, 1995), p. 2889.

[3] David L. Rubin for the CESR Operations Group, in Proceed- ings of the 1995 Particle Accelerator Conference and Interanational Conference on High Energy Accelerators, Dallas, 1995 (IEEE, New York, 1995).

[4] Y. Kubota, et al., CLEO Collaboration, Nucl. Instrum. Methods Phys. Res. A 820, 66 (1990).

[5] W. R. Ross, Nucl. Instrum. Methods Phys. Res. A 386 (1997).

[6] J. T. Seeman, in Nonlinear Dynamics Aspects of Particle Accelerators (Springer-Verlag, Berlin, 1986).

[7] S. Milton (unpublished); D. Rice (private communications). 